\title{
On a Non-cooperative Model for Wavelength Assignment in Multifiber Optical Networks ${ }^{\star}$
}

\author{
Evangelos Bampas, Aris Pagourtzis, George Pierrakos, and Katerina Potika \\ School of Elec. \& Comp. Eng., National Technical University of Athens \\ Polytechnioupoli Zografou, 15780 Athens, Greece \\ \{ebamp,pagour, gpierr, epotik\}@cs.ntua.gr
}

\begin{abstract}
We study path multicoloring games that describe situations in which selfish entities possess communication requests in a multifiber all-optical network. Each player is charged according to the maximum fiber multiplicity that her color (wavelength) choice incurs and the social cost is the maximum player cost. We investigate the price of anarchy of such games and provide two different upper bounds for general graphsnamely the number of wavelengths and the minimum length of a path of maximum disutility, over all worst-case Nash Equilibria - as well as matching lower bounds which hold even for trees; as a corollary we obtain that the price of anarchy in stars is exactly 2 . We also prove constant bounds for the price of anarchy in chains and rings in which the number of wavelengths is relatively small compared to the load of the network; in the opposite case we show that the price of anarchy is unbounded.
\end{abstract}

Keywords: Selfish wavelength assignment, non-cooperative games, price of anarchy, multifiber optical networks, path multicoloring.

\section{Introduction}

The need for efficient access to the optical bandwidth in all-optical networks has given rise to the study of several optimization problems in the past years. The most well-studied among them is the problem of assigning a path and a color (wavelength) to each communication request in such a way that paths of the same color are edge-disjoint and the number of colors used is minimized. Nonetheless, it has become clear that the number of wavelengths in commercially available fibers is rather limited - and will probably remain such in the foreseeable future. Fortunately, the use of multiple fibers has come to the rescue. However, fibers are not unlimited either, therefore it makes sense to minimize their usage. This is particularly interesting from the customer's point of view, for example in

\footnotetext{
* This work has been funded by the project PENED 2003. The project is cofinanced $75 \%$ of public expenditure through EC-European Social Fund, 25\% of public expenditure through Ministry of Development-General Secretariat of Research and Technology of Greece and through private sector, under measure 8.3 of Operational Programme "Competitiveness" in the 3rd Community Support Programme.
} 
situations where one can hire a number of parallel fibers for a certain period and the cost depends on that number.

To this end, several optimization problems have been defined and studied, the objective being to minimize either the maximum fiber multiplicity per edge [12:3 or the sum of these maximum multiplicities over all edges of the graph 4 456]; in another scenario the allowed fiber multiplicity per edge is given and the goal is to minimize the number of wavelengths needed [7/8,5].

In this work we consider a non-cooperative model, where each request is issued by a user who tries to optimize her own fiber usage by selecting the most appropriate wavelength, taking into account other users' choice. This model is mainly motivated by the lack of centralized control in large scale networks. We assume that each user is charged according to the maximum fiber multiplicity that the user's choice incurs. More specifically, a user will be charged according to the maximum number of paths that share an edge with her and use the same wavelength. We consider as social cost the maximum fiber multiplicity that appears on any edge of the network. Minimizing this quantity is particularly important in cases where fibers are hired or sold as a whole, hence the maximum number of fibers needed on an edge determines the total cost; further motivation can be found in papers that address the corresponding optimization problem (see e.g. [ $\left.\begin{array}{lll}1 & 2 & 3\end{array}\right]$ ). Here we focus on situations where routing is unique (acyclic topologies) or pre-determined - as happens in many practical settings, for example in cases where there are specific routing constraints such as a requirement to use lightpaths that have been set in advance, or shortest path routing.

We formulate the above model by defining the class of SELFisH PATH MULTICOLORING (S-PMC) games: the input is a graph, a set of paths, and the number of colors $w$. Each player controls a path in the graph and has to choose a color for that path from $\left\{\alpha_{1}, \ldots, \alpha_{w}\right\}$. A player is charged according to the maximum multiplicity of her color along her path. We consider as social cost the maximum color multiplicity per edge, i.e., the maximum number of paths of same color that use an edge.

Related work. Arguably, the most important notion in the theory of non-cooperative games is the Nash Equilibrium (NE) [9], a stable state of the game in which no player has incentive to change strategy unilaterally. A fundamental question in this theory concerns the existence of pure Nash Equilibria (PNE). For various games 1011,1213 it has been shown that a PNE exists and can usually be found with the use of potential functions. A standard measure of the worst-case quality of Nash Equilibria relative to optimal solutions is the price of anarchy (PoA) 14, which has been extensively studied for load balancing games [14 15] and other problems such as routing and facility location 10 16. A second known measure related to NE is the price of stability (PoS), defined in [17.

$\mathrm{S}-\mathrm{PMC}$ games are closely related to a variation of congestion games [1819 where a player's cost is determined by her maximum latency instead of the usual cost which is the sum of her latencies. Next, we briefly explain the relation of those models to ours. 
In [18] the authors study atomic routing games on networks, where each player chooses a path to route her traffic from an origin to a destination node, with the objective of minimizing the maximum congestion on any edge of her path. They show that these games always possess at least one optimal PNE (hence the PoS is 1) and that the PoA of the game is determined by topological properties of the network; in particular they show that the PoA is upper bounded by the length of the longest path in the player strategy sets and lower bounded by the length of the longest cycle. Some of our results extend to their model, since our model mimics traffic routing in the following sense: we may consider a multigraph, where we replace each edge with $w$ parallel edges, one for each color. Each player's strategy set then consists of $w$ different source-destination paths, corresponding to the $w$ available colors in the original model. A further generalization is the model of Banner and Orda [19, where they introduce the notion of bottleneck games. In this model they allow arbitrary latency functions on the edges and consider both the case of splittable and unsplittable flows. They show existence, convergence and non-uniqueness of equilibria and they prove that the PoA for these games is unbounded. Both models are more general than ours; however our model fits better into the framework of all-optical networks for which we manage to provide, among others, smaller upper bounds on the PoA compared to the ones obtained by [1819], as well as a better convergence rate to Nash equilibria. In [20] they study similar games and give results for restricted cases, e.g. single-commodity networks.

To the best of our knowledge selfish path multicoloring games have not been studied before. Selfish path coloring in single fiber all-optical networks have been studied in 21/22 2324]. Bilò and Moscardelli [21] consider the convergence to Nash Equilibria of selfish routing and path coloring games. Later, Bilò et al. 22] considered different information levels of local knowledge that players may have for computing their payments in the same games and give bounds for the PoA in chains, rings and trees. The existence of Nash Equilibria and the complexity of recognizing and computing a Nash Equilibrium for selfish routing and path colorings games under several payment functions are considered by Georgakopoulos et al. 23. In 24] upper and lower bounds of the PoA for selfish path coloring with and without routing are presented under functions that charge a player only according to her own strategy.

Our results. We first give an upper bound on the convergence rate of Nash dynamics for S-PMC games, and observe that the price of stability is always equal to 1. We also show how to efficiently compute a Nash Equilibrium of minimum social cost for S-PMC games in rooted trees, i.e. trees in which each path lies entirely on a simple path from some fixed root node to a leaf. For S-PMC games in stars, we prove that a known approximation algorithm for a related optimization problem actually gives an $\frac{1}{2}$-approximate Nash Equilibrium.

For general graphs, we obtain two upper bounds on the PoA: the first, which is not hard to show, is equal to the number of available colors. The second, which requires more involved arguments, is equal to the length of a shortest 
path with maximum disutility in any worst-case NE. For both bounds we provide matching lower bounds. In fact, we prove that these bounds hold even in trees.

Then, we move on to specific network topologies and show that for S-PMC games in stars $\mathrm{PoA}=2$. We also provide constant bounds on the PoA in a broad class of S-PMC games in chains and rings, namely for all games with $L=\Omega\left(w^{2}\right)$, where $w$ is the number of available colors and $L$ is the maximum load among all edges of the network. On the other hand, for any $\varepsilon>0$ we exhibit a class of S-PMC games in chains (and rings) with $L=\Theta\left(w^{2-\varepsilon}\right)$ for which the PoA is unbounded.

In order to show our upper bounds, we demonstrate path patterns that must be present in any Nash Equilibrium, while for the lower bounds we employ recursive construction techniques.

\section{Definitions and Model}

Given an undirected graph $G(V, E)$, a set $P$ of simple paths defined on $G$, and a set $W=\left\{\alpha_{1}, \ldots, \alpha_{w}\right\}$ of available colors, $L(e)$ will denote the load of edge $e$, i.e. the number of paths that use edge $e$. The maximum of these loads will be denoted by $L$, i.e. $L=\max _{e \in E} L(e)$.

Given, additionally, an assignment of a color to each path we define the following:

\section{Definition 1}

1. $\mu(e, c)$ will denote the multiplicity of color $c$ on edge e, i.e. the number of paths that use edge $e$ and are colored with color $c$.

2. $\mu_{e}$ will denote the maximum multiplicity of any color on edge e, i.e. $\mu_{e}=$ $\max _{c \in W} \mu(e, c)$.

3. $\mu_{\max }$ will denote the maximum multiplicity of any color over all edges: $\mu_{\max }=$ $\max _{e \in E} \mu_{e}$.

4. $\mu(p, c)$ will denote the maximum multiplicity of color $c$ over the edges of path $p: \mu(p, c)=\max _{e \in p} \mu(e, c)$.

It will be clear from the context which specific coloring we are referring to when we use the above notation.

The minimum $\mu_{\max }$ that can be attained by some coloring of the paths in $P$ will be denoted by $\mu_{\mathrm{OPT}}$, i.e. $\mu_{\mathrm{OPT}}=\min _{\boldsymbol{c}} \mu_{\max }$ where $\boldsymbol{c}$ ranges over all possible colorings. We note immediately the following:

Fact. No coloring can achieve a $\mu_{\max }$ smaller than $\left\lceil\frac{L}{w}\right\rceil$. Thus, $\mu_{\mathrm{OPT}} \geq\left\lceil\frac{L}{w}\right\rceil$.

We now proceed to define the class of selfish path multicoloring games and subclasses thereof.

Definition 2 (Selfish path multicoloring games). A selfish path multicoloring game is the following strategic game defined in terms of an undirected graph $G$, a set $P$ of simple paths defined on $G$, and an integer $w>0$ : 
- Players: there is one player for each path in P. For simplicity, we identify a player $i$ with the corresponding path $p_{i}$.

- Strategies: a strategy for player $i$ is a color $c_{i}$ chosen from the set $W=$ $\left\{\alpha_{1}, \ldots, \alpha_{w}\right\}$ of available colors. We say that color $c_{i}$ is assigned to path $p_{i}$ or that path $p_{i}$ is colored with color $c_{i}$. All players share the common set of strategies $W$.

- Disutility: given a strategy profile $\boldsymbol{c}=\left(c_{1}, \ldots, c_{|P|}\right)$, the disutility $f_{i}: W^{|P|} \rightarrow$ $\mathbb{N}$ of each player $i$ is defined as follows:

$$
f_{i}(\boldsymbol{c})=\mu\left(p_{i}, c_{i}\right) .
$$

We denote this game by $\langle G, P, w\rangle$. The class of all selfish path multicoloring games will be denoted by S-PMC.

We will use the notation $\mathrm{S}-\mathrm{PMC}(\mathcal{G})$ to denote a subclass of S-PMC that contains only games satisfying a property $\mathcal{G}$ (for example $\mathcal{G}$ may constrain the graph on which the game is defined to belong to a specific graph class, etc.).

Following the standard definition, a strategy profile $\boldsymbol{c}=\left(c_{1}, \ldots, c_{|P|}\right)$ is said to be a pure Nash Equilibrium (PNE), or simply Nash Equilibrium (NE), if for each player $i$ it holds that: $f_{i}\left(c_{1}, \ldots, c_{i}^{\prime}, \ldots, c_{|P|}\right) \geq f_{i}\left(c_{1}, \ldots, c_{i}, \ldots, c_{|P|}\right)$, for any strategy $c_{i}^{\prime} \in W$. Moreover, following the definition of [25], we say that a strategy profile $\boldsymbol{c}=\left(c_{1}, \ldots, c_{|P|}\right)$ is an $\varepsilon$-approximate Nash Equilibrium if for each player $i$ it holds that: $f_{i}\left(c_{1}, \ldots, c_{i}^{\prime}, \ldots, c_{|P|}\right) \geq(1-\varepsilon) \cdot f_{i}\left(c_{1}, \ldots, c_{i}, \ldots, c_{|P|}\right)$, for any strategy $c_{i}^{\prime} \in W$.

Definition 3 (Blocking edges). If $\boldsymbol{c}$ is a strategy profile for a game $\langle G, P, w\rangle$ and $p_{i} \in P$, we say that edge $e$ is an $\alpha_{j}$-blocking edge for $p_{i}$, or that it blocks $\alpha_{j}$ for $p_{i}$, if $e \in p_{i}$ and $\mu\left(e, \alpha_{j}\right) \geq f_{i}(\boldsymbol{c})-1$. Furthermore, the $\mu\left(e, \alpha_{j}\right)$ paths that are colored with $\alpha_{j}$ and use edge $e$ are called $\alpha_{j}$-blocking paths for $p_{i}$.

Intuitively, an $\alpha_{j}$-blocking edge for $p_{i}$ "blocks" $p_{i}$ from switching to color $\alpha_{j}$ because if it did, the new disutility of path $p_{i}$ would be at least $\mu\left(e, \alpha_{j}\right)+1 \geq$ $f_{i}(\boldsymbol{c})$, no better than its current choice. It is immediate from the definitions that the following property holds in any Nash Equilibrium of any S-PMC game:

Property 1 (Structural property of S-PMC Nash Equilibria). In a Nash Equilibrium, every path $p$ must contain at least one $\alpha_{j}$-blocking edge for $p$, for every color $\alpha_{j}$.

Definition 4 (Social cost). The social cost of a strategy profile $\boldsymbol{c}$ for an $\mathrm{S}$ PMC game is defined as follows: $\operatorname{sc}(\boldsymbol{c})=\max _{e \in E} \mu_{e}=\mu_{\max }$.

It is straightforward to verify that the social cost of a strategy profile coincides with the maximum player disutility in that profile:

$$
\operatorname{sc}(\boldsymbol{c})=\max _{e \in E} \mu_{e}=\max _{p_{i} \in P} f_{i}(\boldsymbol{c})
$$

We define $\hat{\mu}$ to be the maximum social cost over all strategy profiles that are Nash Equilibria: $\hat{\mu}=\max _{\boldsymbol{c}}$ is $\mathrm{NE} \operatorname{sc}(\boldsymbol{c})$. Following the standard definitions, the 
price of anarchy (PoA) of a game $\langle G, P, w\rangle$ is the worst-case social cost in a Nash Equilibrium divided by $\mu_{\mathrm{OPT}}$, i.e.: $\operatorname{PoA}(\langle G, P, w\rangle)=\frac{\max _{\boldsymbol{c}} \text { is } \mathrm{NE} \operatorname{sc}(\boldsymbol{c})}{\mu_{\mathrm{OPT}}}=\frac{\hat{\mu}}{\mu_{\mathrm{OPT}}}$. The price of stability $(\mathrm{PoS})$ of a game is the best-case social cost in a NE divided by $\mu_{\mathrm{OPT}}: \operatorname{PoS}(\langle G, P, w\rangle)=\frac{\min _{c \text { is NE }} \operatorname{sc}(\boldsymbol{c})}{\mu_{\mathrm{OPT}}}$. The price of anarchy (resp. stability) of a class of games $\mathrm{S}-\mathrm{PMC}(\mathcal{G})$ is the maximum price of anarchy (resp. stability) among all games in $\mathrm{S}-\mathrm{PMC}(\mathcal{G})$.

\section{Existence and Computation of Nash Equilibria}

We use lexicographic-order arguments similar to those in 1819] to show that in any S-PMC game the following holds: starting from an arbitrary strategy profile any Nash dynamics converges to a Nash Equilibrium of smaller or equal social cost. The proof is omitted.

Theorem 1. For any game $\langle G, P, w\rangle$ in S-PMC:

a. the price of stability is 1 , and

b. any Nash dynamics converges to a Nash Equilibrium in at most $4^{|P|}$ steps.

Due to Theorem 1, computing a Nash Equilibrium of minimum social cost is at least as hard as the corresponding optimization problem. As noticed in [4] this problem is NP-hard in general graphs, in fact even in rings and stars. Therefore, it is also NP-hard to compute an optimal Nash Equilibrium even in the case of rings and stars. However, we show that there exists an efficient algorithm that computes optimal Nash Equilibria for a subclass of S-PMC(TrEE). Furthermore, we show that we can use a known algorithm for PATH MultiColoring in stars [4] to compute approximate Nash Equilibria for S-PMC(STAR) games. We will only state the theorems and omit the proofs.

Definition 5. We define S-PMC(RoOTED-TREE) to be the subclass of SPMC that contains games $\langle G, P, w\rangle$ with the following property: " $G$ is a tree and there is a node $r$ such that each path in $P$ lies entirely on some simple path from $r$ to a leaf."

Consider the greedy algorithm that colors paths in order of non-decreasing distance from the root in such a way that the color multiplicity is the lowest possible with respect to the current partial coloring.

Theorem 2. Given an S-PMC(Rooted-Tree) game $\langle G(V, E), P, w\rangle$ with maximum load $L$ as input, the greedy algorithm computes an optimal Nash Equilibrium of cost exactly $\left\lceil\frac{L}{w}\right\rceil$.

Theorem 3. There is a polynomial-time algorithm that computes a $\frac{1}{2}$-approximate Nash Equilibrium for any S-PMC(STAR) game. 


\section{Tight Upper Bounds for the PoA of S-PMC Games}

In this section we provide two upper bounds on the PoA of any S-PMC game and we show that both of them are tight. The first bound is determined by a property of the network, namely the number of available wavelengths. The second bound is more subtle, as it depends on the length of paths with the highest disutility in worst-case Nash Equilibria. We prove that these bounds are tight even for the class S-PMC(Rooted-TreE), and asymptotically tight for the class SPMC(Rooted-Tree: $\Delta=3$ ), i.e. the subclass of S-PMC(Rooted-Tree) that contains games defined on graphs with maximum degree 3 .

Lemma 1. The price of anarchy of any $\mathrm{S}-\mathrm{PMC}$ game $\langle G, P, w\rangle$ is at most $w$.

Proof. Let $\boldsymbol{c}$ be a worst-case Nash Equilibrium of $\langle G, P, w\rangle$, hence $\operatorname{sc}(\boldsymbol{c})=\hat{\mu}$. Clearly, $\hat{\mu} \leq L$ and since the minimum social cost over all strategy profiles is $\mu_{\mathrm{OPT}} \geq\left\lceil\frac{L}{w}\right\rceil$, it turns out that $\mu_{\mathrm{OPT}} \geq \frac{\hat{\mu}}{w}$. This implies that $\frac{\hat{\mu}}{\mu_{\mathrm{OPT}}} \leq w$.

Lemma 2. For any worst-case Nash Equilibrium c of an S-PMC game $\langle G, P, w\rangle$ and for any $p_{i} \in P$ with $f_{i}(\boldsymbol{c})=\operatorname{sc}(\boldsymbol{c})=\hat{\mu}$, the price of anarchy of $\langle G, P, w\rangle$ is at most equal to the length of $p_{i}$.

Proof. Let $\tilde{e}$ be an edge of $p_{i}$ where the color $c_{i}$ chosen by $p_{i}$ appears with maximum multiplicity $\hat{\mu}: \mu\left(\tilde{e}, c_{i}\right)=\hat{\mu}$. Let $z$ denote the length of path $p_{i}$ and let $e_{1}, \ldots, e_{z-1}$ be the edges that $p$ uses, apart from $\tilde{e}$. For $1 \leq j \leq z-1$, let $x_{j}$ be the number of colors that are blocked for $p_{i}$ on $e_{j}$ and let $y$ be the number of colors that are blocked for $p_{i}$ on $\tilde{e}$ (since $\boldsymbol{c}$ is a Nash Equilibrium, it must be that $\left.x_{1}+\ldots+x_{z-1}+y \geq w-1\right)$.

If it is the case that $z=1$, i.e. $p_{i}$ uses only edge $\tilde{e}$, then $\tilde{e}$ must block all colors for $p_{i}$ except $c_{i}$. This implies that the load of edge $\tilde{e}$ is: $L(\tilde{e}) \geq \hat{\mu}+(w-1)(\hat{\mu}-1)=$ $w \hat{\mu}-w+1$. Therefore, the minimum social cost over all strategy profiles satisfies: $\mu_{\mathrm{OPT}} \geq\left\lceil\frac{L(\tilde{e})}{w}\right\rceil \geq\left\lceil\hat{\mu}-\frac{w-1}{w}\right\rceil=\hat{\mu}$. We conclude that the price of anarchy in this case is equal to 1 .

Now, assume that $z \geq 2$. We will prove that $L \geq 1+\left\lceil\frac{w}{z}\right\rceil(\hat{\mu}-1)$. First, observe that $L(\tilde{e}) \geq \hat{\mu}+y(\hat{\mu}-1)$ and, for $1 \leq j \leq z-1, L\left(e_{j}\right) \geq 1+x_{j}(\hat{\mu}-1)$. If $y \geq\left\lceil\frac{w}{z}\right\rceil-1$, then $L(\tilde{e}) \geq \hat{\mu}+\left(\left\lceil\frac{w}{z}\right\rceil-1\right)(\hat{\mu}-1)=1+\left\lceil\frac{w}{z}\right\rceil(\hat{\mu}-1)$, therefore $L \geq 1+\left\lceil\frac{w}{z}\right\rceil(\hat{\mu}-1)$. If, on the other hand, $y<\left\lceil\frac{w}{z}\right\rceil-1$, then $x_{1}+\ldots+$ $x_{z-1} \geq w-1-y \geq w-\left\lceil\frac{w}{z}\right\rceil+1$. This implies that there is some $x_{k}$ such that $x_{k} \geq \frac{w-\left\lceil\frac{w}{z}\right\rceil+1}{z-1}>\frac{w-\frac{w}{z}-1+1}{z-1}=\frac{w}{z}$. Since $x_{k}$ is an integer, it must be that $x_{k} \geq\left\lceil\frac{w}{z}\right\rceil$. Therefore, $L \geq L\left(e_{k}\right) \geq 1+\left\lceil\frac{w}{z}\right\rceil(\hat{\mu}-1)$.

We conclude that in any case $L \geq 1+\left\lceil\frac{w}{z}\right\rceil(\hat{\mu}-1)$. So, the price of anarchy is bounded as follows:

$$
\operatorname{PoA}(\langle G, P, w\rangle)=\frac{\hat{\mu}}{\mu_{\mathrm{OPT}}} \leq \frac{\hat{\mu}}{\left\lceil\frac{L}{w}\right\rceil} \leq \frac{\hat{\mu}}{\left\lceil\frac{1+\left\lceil\frac{w}{z}\right\rceil(\hat{\mu}-1)}{w}\right\rceil} \leq z .
$$

We omit the proof of the last inequality, which holds for all $\hat{\mu} \geq 2, w \geq 1$, and $z \geq 2$. 


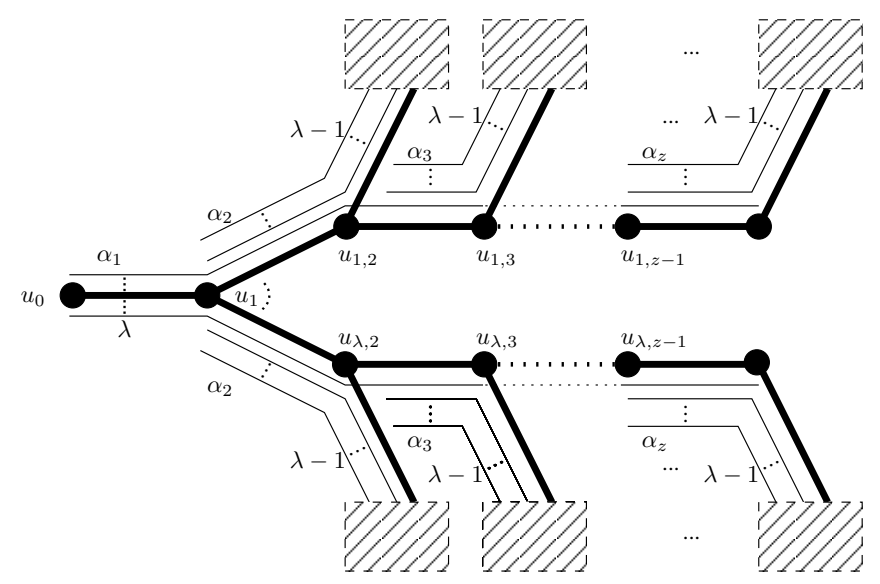

Fig. 1. The construction $A_{z}(\lambda)$ for the proof of Lemma 3 The thick lines represent the edges of the underlying graph, and the thin lines represent the paths defined on the graph. The color and multiplicity of each group of paths is written next to that group. Each shaded box represents a recursive copy of $A_{z}(\lambda-1)$.

As an immediate corollary of Lemma 2, we derive the following upper bound on the price of anarchy:

Corollary 1. The price of anarchy of any S-PMC game $\langle G, P, w\rangle$ is bounded as follows:

$$
\mathrm{PoA} \leq \min _{\boldsymbol{c}: \mathrm{NE} \wedge \mathrm{sc}(\boldsymbol{c})=\hat{\mu}} \min _{i: f_{i}(\boldsymbol{c})=\hat{\mu}} \operatorname{length}\left(p_{i}\right)
$$

Lemma 3. The upper bounds of Lemma 1 and Corollary 1 are tight even for the class of S-PMC(RoOTED-TREe) games.

Proof. We first define a recursive construction of an S-PMC game and a Nash Equilibrium for this game. The construction is illustrated in Figure 1 For any $z \geq 1$ and $\lambda \geq 1$, let $A_{z}(\lambda)$ be the following S-PMC game with $z$ available colors: there are $\lambda$ paths of color $\alpha_{1}$ and length $z$, starting at the "root node" $u_{0}$, which branch out into $\lambda$ branches, one on each branch. Let us call these the "primary" paths for $A_{z}(\lambda)$. On any of the $z-1$ edges of each such branch, one color is blocked for the primary path. The $\lambda-1$ blocking paths of each edge branch out into an $A_{z}(\lambda-1)$ game. They become primary paths for this copy of $A_{z}(\lambda-1)$. The root node for the $j$-th recursive copy of $A_{z}(\lambda-1)$ on the $k$-th branch is node $u_{k, j}$ (node $u_{k, 1}$ is common for all branches). The base case of this recursive construction is $A_{z}(0)$, which is a degenerate game with no paths and no available colors, defined on a graph consisting of a single node.

Observe that for any $z \geq 1$, the construction $A_{z}(z)$ is an S-PMC(RootedTrEe) game in NE, in which all of the following are equal to $z: w, L, \mu_{\max }$, and all path lengths. By Theorem 2, the optimal strategy profile for $A_{z}(z)$ has social cost $\mu_{\mathrm{OPT}}=\left\lceil\frac{L}{w}\right\rceil=1$. Therefore, the ratio $\frac{\mu_{\max }}{\mu_{\mathrm{OPT}}}$ is equal to $z$ for this Nash Equilibrium, hence the price of anarchy is at least $z$. 
By appropriate modification of the construction presented in Figure 1, we obtain the following:

Lemma 4. The upper bounds of Lemma 1 and Corollary 1 are asymptotically tight even for the class of S-PMC(ROOTED-TREE) games with maximum degree 3 .

We summarize the results of Lemmata 1, 2, 3, and 4] in the following theorem:

Theorem 4. The price of anarchy of any S-PMC game $\langle G, P, w\rangle$ is upperbounded both by $w$ and by

$$
\min _{\boldsymbol{c}: \mathrm{NE} \wedge \mathrm{sc}(\boldsymbol{c})=\hat{\mu}} \min _{i: f_{i}(\boldsymbol{c})=\hat{\mu}} \text { length }\left(p_{i}\right) .
$$

These bounds are tight for the class S-PMC(ROOTED-TREE) and asymptotically tight for the class S-PMC(Rooted-TREE: $\Delta=3$ ).

Theorem 5. The price of anarchy of the class $\mathrm{S}-\mathrm{PMC}(\mathrm{STAR})$ is 2.

Proof. Lemma 2 implies an upper bound of 2 on the price of anarchy since the length of any path in a star cannot be larger than 2 .

For the lower bound, it can be shown that the construction of Lemma 3 can be modified to yield a family of S-PMC(STAR) games with price of anarchy 2. More specifically every game $A_{2}(\lambda)$ can be embedded in a star, by using additional star rays for branching. The detailed construction is omitted.

\section{The Price of Anarchy on Graphs of Maximum Degree 2}

In this section we study the price of anarchy of path multicoloring games on chains and rings, and we prove a constant upper bound for a broad class of S-PMC(RING) games with $L=\Omega\left(w^{2}\right)$. Notice that this class essentially encompasses all S-PMC(RING) games of practical importance, as the number of wavelengths is limited in practice due to technological constraints, whereas $L$ can grow large depending on network traffic. For the sake of completeness, we show that the PoA becomes quickly unbounded if we allow the network designer to provide ample wavelengths to the users, i.e. when $L=o\left(w^{2}\right)$.

We begin by strengthening Property 1 to prove a more involved structural property of Nash Equilibria in S-PMC(RING) games. Let $\langle G, P, w\rangle$ be an $\mathrm{S}$ PMC(RInG) game. Given a coloring $\boldsymbol{c}=\left(c_{1}, \ldots, c_{|P|}\right)$, let $P\left(e, \alpha_{i}\right)(\boldsymbol{c}) \subseteq P$ denote the set of paths colored with color $\alpha_{i}$ that use edge $e \in E$; by definition $\left|P\left(e, \alpha_{i}\right)(\boldsymbol{c})\right|=\mu\left(e, \alpha_{i}\right)$. For the sake of simplicity, in the rest of the section we will write $P\left(e, \alpha_{i}\right)$ instead of $P\left(e, \alpha_{i}\right)(\boldsymbol{c})$. Furthermore, let $\left[e_{l}, e_{r}\right]$ denote the clockwise arc starting at edge $e_{l}$ and ending at edge $e_{r}$.

Lemma 5 (Structural property of S-PMC(Ring) NE). Given a game in $\mathrm{S}-\mathrm{PMC}(\mathrm{RING})$ and a coloring $\boldsymbol{c}$ thereof which is a Nash Equilibrium, for every edge $e$ and color $\alpha_{i}$ there is an edge-simple arc $\left[e_{l}, e_{r}\right]$ with the following properties: 
a. for every color $\alpha_{j} \neq \alpha_{i}$, arc $\left[e_{l}, e_{r}\right]$ contains an edge which is an $\alpha_{j}$-blocking edge for at least half of the paths in $P\left(e, \alpha_{i}\right)$, and

b. for every edge $e^{\prime}$ of the arc $\left[e_{l}, e_{r}\right]$ it holds that $\left|P\left(e^{\prime}, \alpha_{i}\right) \cap P\left(e, \alpha_{i}\right)\right| \geq$ $\left\lceil\frac{\left|P\left(e, \alpha_{i}\right)\right|}{2}\right\rceil$.

Proof. Since the game is in NE, by Property 1 every path $p \in P\left(e, \alpha_{i}\right)$ must have at least one $\alpha_{j}$-blocking edge, for every color $\alpha_{j} \neq \alpha_{i}$. For a fixed color $\alpha_{j} \neq \alpha_{i}$, consider the two $\alpha_{j}$-blocking edges for some path in $P\left(e, \alpha_{i}\right)$ that are closest to edge $e$ clockwise and counter-clockwise. It is not hard to see that for at least one of these two edges, call it $b\left(\alpha_{j}\right)$, the following property holds: the arc $\left[e, b\left(\alpha_{j}\right)\right]$ or the arc $\left[b\left(\alpha_{j}\right), e\right]$ is contained in at least $\left[\frac{\left|P\left(e, \alpha_{i}\right)\right|}{2}\right]$ of the paths in $P\left(e, \alpha_{i}\right)$. In case that there is only one $\alpha_{j}$-blocking edge for all paths in $P\left(e, \alpha_{i}\right)$, then the property holds a fortiori for this edge.

For every color $\alpha_{j}$ we pick one such edge $b\left(\alpha_{j}\right)$. If the above property holds for arc $\left[e, b\left(\alpha_{j}\right)\right]$, we add $b\left(\alpha_{j}\right)$ to set $B^{+}$, otherwise we add it to set $B^{-}$. We now claim that a clockwise traversal of the ring starting at edge $e$ will first encounter all edges of $B^{+}$and then all edges of $B^{-}$. Indeed, if one edge $b^{-}$of $B^{-}$lies before one edge $b^{+}$of $B^{+}$on this clockwise traversal, this would imply that $b^{-}$is traversed by the $\left\lceil\frac{\left|P\left(e, \alpha_{i}\right)\right|}{2}\right\rceil$ paths that contain the arc $\left[e, b^{+}\right]$and thus $b^{-}$should also belong to $B^{+}$.

The above discussion implies that if we define $e_{r}$ to be the last edge of $B^{+}$ and $e_{l}$ to be the first edge of $B^{-}$encountered in this clockwise traversal, then the edge-simple arc $\left[e_{l}, e_{r}\right]$ satisfies the conditions of the Lemma.

We now prove a constant upper bound on the price of anarchy of S-PMC(RING) games with $L=\Omega\left(w^{2}\right)$; denote this class by S-PMC(RING: $\left.L=\Omega\left(w^{2}\right)\right)$. This also provides an upper bound on the price of anarchy of any S-PMC(CHAIN: $\left.L=\Omega\left(w^{2}\right)\right)$ game, as every game defined on a chain can be trivially embedded in a ring topology.

We first employ the structural property of S-PMC(RING) Nash Equilibria (Lemma 5) in order to establish the existence of a heavily loaded edge in SPMC(RING) games with $\hat{\mu} \geq w$.

Lemma 6. In every $\mathrm{S}-\mathrm{PMC}(\mathrm{RING})$ game $\langle G, P, w\rangle$ with $\hat{\mu} \geq w$ there is an edge with load at least $\frac{\hat{\mu} w}{4}$.

Proof. Let $\left[e_{l}, e_{r}\right]_{P\left(e, \alpha_{i}\right)}$ be the arc that is obtained by applying Lemma 5 for path set $P\left(e, \alpha_{i}\right)$. We define $P_{1}$ to be the set of paths $P\left(\tilde{e}, \alpha_{1}\right)$ which induce the social cost $\hat{\mu}$. For $i \geq 2$ we define $P_{i}$ to be the set of $\alpha_{j}$-blocking paths for the path set $P_{i-1}$, for some color $\alpha_{j}$ not appearing at any of the path sets $P_{k}, k<i$, with the following property:

$$
\left[e_{l}, e_{r}\right]_{P_{i}} \subseteq\left[e_{l}, e_{r}\right]_{P_{i-1}},
$$

if such a path set exists. If more than one path sets with the desired property exist, we arbitrarily pick one of them.

Let $e_{i}$ be the $\alpha_{j}$-blocking edge for $P_{i-1}$; based on the inductive definition of $P_{i}$ as a set of blocking paths for path set $P_{i-1}$ we can easily show that $\mu\left(e_{i}, \alpha_{j}\right) \geq$ 
$\hat{\mu}-i+1$. Applying Lemma [5(b) for color $\alpha_{j}$ and edge $e_{i}$ yields the following: for every edge $e \in\left[e_{l}, e_{r}\right]_{P_{i}}$ we have that $\mu\left(e, \alpha_{j}\right) \geq \frac{\hat{\mu}-i+1}{2}$. Furthermore, since Equation 1 holds for all $k \leq i$, the load of all edges $e \in\left[e_{l}, e_{r}\right]_{P_{i}}$ is at least $\sum_{\alpha_{j}} \mu\left(e, \alpha_{j}\right)$, where $\alpha_{j}$ now ranges over the colors of all path sets $P_{k}, k \leq i$. Hence, for every edge $e \in\left[e_{l}, e_{r}\right]_{P_{i}}$ we have that $L(e) \geq \sum_{\alpha_{j}} \mu\left(e, \alpha_{j}\right) \geq \sum_{k=1}^{i} \frac{\hat{\mu}-k+1}{2}$.

Let now $n$ be the first integer for which no such path set $P_{n}$ exists and consider the path set $P_{n-1}$. Since we are at Nash Equilibrium we know that there exist $\alpha$-blocking edges for paths in $P_{n-1}$, for every color $\alpha$. We restrict our attention to the $w-n+1$ colors, which have not yet appeared at any $P_{k}$, for $k \leq n-1$; let $\alpha_{j}$ be one of these colors. Consider now an $\alpha_{j}$-blocking edge $e_{n}$ such that $e_{n} \in\left[e_{l}, e_{r}\right]_{P_{n-1}}$ (by Lemma[5(a) such an edge must exist). We now have that, at least half of the $\alpha_{j}$-blocking paths in $P\left(e_{n}, \alpha_{j}\right)$, i.e. at least $\frac{\hat{\mu}-n+1}{2}$ paths, extend beyond one of the edges $e_{l}\left(P_{n-1}\right), e_{r}\left(P_{n-1}\right)$ of the arc $\left[e_{l}, e_{r}\right]_{P_{n-1}}$ (otherwise we would have picked $P\left(e_{n}, \alpha_{j}\right)$ to be $\left.P_{n}\right)$. This means that for at least half of these $w-n+1$ blocking path sets, their paths leave the arc from the same edge, incurring on it an additional load of $\frac{w-n+1}{2} \cdot \frac{\hat{\mu}-n+1}{2}$.

Thus, the total load of this edge is at least $\sum_{i=1}^{n-1} \frac{\hat{\mu}-i+1}{2}+\frac{w-n+1}{2} \cdot \frac{\hat{\mu}-n+1}{2}=$ $\frac{\hat{\mu} w}{4}+(n-1) \cdot \frac{\hat{\mu}-w+1}{4}$. Since $\hat{\mu} \geq w$ the above sum is at least $\frac{\hat{\mu} w}{4}$.

Theorem 6. The price of anarchy of any game in the class S-PMC(RING: $\left.L=\Omega\left(w^{2}\right)\right)$ is bounded by a constant.

Proof. We distinguish between two cases:

- If $\hat{\mu} \geq w$, then by Lemma 6 we get $L \geq \frac{\hat{\mu} w}{4}$. This implies $\frac{L}{w} \geq \frac{\hat{\mu}}{4} \Rightarrow \mu_{\mathrm{OPT}} \geq$ $\frac{\hat{\mu}}{4} \Rightarrow \mathrm{PoA} \leq 4$.

- If $\hat{\mu}<w$, then PoA $=\frac{\hat{\mu}}{\mu_{\mathrm{OPT}}} \leq \frac{\hat{\mu} w}{L}<\frac{w^{2}}{L}$, where we used successively the facts that $\mu_{\mathrm{OPT}} \geq \frac{L}{w}$ and $\hat{\mu}<w$. The last inequality, combined with the fact that $L=\Omega\left(w^{2}\right)$, implies PoA $=O(1)$.

Finally, we show that the price of anarchy can get arbitrarily large when the number of available colors increases; specifically, that it is unbounded for the classes S-PMC(ChAin: $\left.L=o\left(w^{2}\right)\right)$ and S-PMC(RInG: $\left.L=o\left(w^{2}\right)\right)$. The proof is omitted.

Theorem 7. For any fixed $\varepsilon>0$ there exists an infinite family of games in S-PMC(CHAIn: $\left.L=\Theta\left(w^{2-\varepsilon}\right)\right)$ with $\operatorname{PoA}=\Omega\left(w^{\frac{\varepsilon}{2}}\right)$.

\section{References}

1. Andrews, M., Zhang, L.: Minimizing maximum fiber requirement in optical networks. J. Comput. Syst. Sci. 72(1), 118-131 (2006)

2. Andrews, M., Zhang, L.: Complexity of wavelength assignment in optical network optimization. In: INFOCOM 2006. IEEE, Los Alamitos (2006)

3. Andrews, M., Zhang, L.: Wavelength assignment in optical networks with fixed fiber capacity. In: Díaz, J., Karhumäki, J., Lepistö, A., Sannella, D. (eds.) ICALP 2004. LNCS, vol. 3142, pp. 134-145. Springer, Heidelberg (2004)

4. Nomikos, C., Pagourtzis, A., Zachos, S.: Routing and path multicoloring. Inf. Process. Lett. 80(5), 249-256 (2001) 
5. Erlebach, T., Pagourtzis, A., Potika, K., Stefanakos, S.: Resource allocation problems in multifiber WDM tree networks. In: Bodlaender, H.L. (ed.) WG 2003. LNCS, vol. 2880, pp. 218-229. Springer, Heidelberg (2003)

6. Winkler, P., Zhang, L.: Wavelength assignment and generalized interval graph coloring. In: SODA, pp. 830-831 (2003)

7. Margara, L., Simon, J.: Wavelength assignment problem on all-optical networks with $k$ fibres per link. In: Welzl, E., Montanari, U., Rolim, J.D.P. (eds.) ICALP 2000. LNCS, vol. 1853, pp. 768-779. Springer, Heidelberg (2000)

8. Li, G., Simha, R.: On the wavelength assignment problem in multifiber WDM star and ring networks. IEEE/ACM Trans. Netw. 9(1), 60-68 (2001)

9. Nash, J.: Non-cooperative games. The Annals of Mathematics 54(2), 286-295 (1951)

10. Fotakis, D., Kontogiannis, S.C., Koutsoupias, E., Mavronicolas, M., Spirakis, P.G.: The structure and complexity of Nash equilibria for a selfish routing game. In: Widmayer, P., Triguero, F., Morales, R., Hennessy, M., Eidenbenz, S., Conejo, R. (eds.) ICALP 2002. LNCS, vol. 2380, pp. 123-134. Springer, Heidelberg (2002)

11. Rosenthal, R.W.: A class of games possessing pure-strategy Nash equilibria. Int. J. Game Theory 2, 65-67 (1973)

12. Milchtaich, I.: Congestion games with player-specific payoff functions. Games and Economic Behavior 13, 111-124 (1996)

13. Monderer, D., Shapley, L.S.: Potential games. Games and Economic Behavior 14, 124-143 (1996)

14. Koutsoupias, E., Papadimitriou, C.H.: Worst-case equilibria. In: Meinel, C., Tison, S. (eds.) STACS 1999. LNCS, vol. 1563, pp. 404-413. Springer, Heidelberg (1999)

15. Mavronicolas, M., Spirakis, P.G.: The price of selfish routing. In: STOC, pp. 510 519 (2001)

16. Roughgarden, T., Tardos, É.: How bad is selfish routing? J. ACM 49(2), 236-259 (2002)

17. Anshelevich, E., Dasgupta, A., Kleinberg, J.M., Tardos, É., Wexler, T., Roughgarden, T.: The price of stability for network design with fair cost allocation. In: FOCS, pp. 295-304. IEEE Computer Society, Los Alamitos (2004)

18. Busch, C., Magdon-Ismail, M.: Atomic routing games on maximum congestion. In: Cheng, S.-W., Poon, C.K. (eds.) AAIM 2006. LNCS, vol. 4041, pp. 79-91. Springer, Heidelberg (2006)

19. Banner, R., Orda, A.: Bottleneck routing games in communication networks. In: INFOCOM 2006. IEEE, Los Alamitos (2006)

20. Caragiannis, I., Galdi, C., Kaklamanis, C.: Network load games. In: Deng, X., Du, D.-Z. (eds.) ISAAC 2005. LNCS, vol. 3827, pp. 809-818. Springer, Heidelberg (2005)

21. Bilò, V., Moscardelli, L.: The price of anarchy in all-optical networks. In: Kralovic, R., Sýkora, O. (eds.) SIROCCO 2004. LNCS, vol. 3104, pp. 13-22. Springer, Heidelberg (2004)

22. Bilò, V., Flammini, M., Moscardelli, L.: On Nash equilibria in non-cooperative alloptical networks. In: Diekert, V., Durand, B. (eds.) STACS 2005. LNCS, vol. 3404, pp. 448-459. Springer, Heidelberg (2005)

23. Georgakopoulos, G.F., Kavvadias, D.J., Sioutis, L.G.: Nash equilibria in all-optical networks. In: Deng, X., Ye, Y. (eds.) WINE 2005. LNCS, vol. 3828, pp. 1033-1045. Springer, Heidelberg (2005)

24. Milis, I., Pagourtzis, A., Potika, K.: Selfish routing and path coloring in all-optical networks. In: Janssen, J., Prałat, P. (eds.) CAAN 2007. LNCS, vol. 4852, pp. 71-84. Springer, Heidelberg (2007)

25. Chien, S., Sinclair, A.: Convergence to approximate Nash equilibria in congestion games. In: SODA, pp. 169-178. SIAM, Philadelphia (2007) 